\title{
EFFECT OF SPACE CHARGE ON STABILITY OF BEAM DISTRIBUTION IN THE SNS RING *
}

\author{
A.V. Fedotov, J. Wei \\ BNL, Upton, NY 11973, USA \\ R.L. Gluckstern \\ University of Maryland, College Park, MD 20742, USA
}

\section{Abstract}

In the Spallation Neutron Source (SNS) ring, multi-turn injection is employed to obtain a large transverse beam size which significantly reduces the space-charge tune shift of the accumulated beam. Careful choice of the painting scheme and bump function is required to obtain the desired beam profile together with low beam loss. In this paper we examine, both analytically and numerically, the effect of the space charge on the beam profile during multi-turn injection painting.

\section{INTRODUCTION}

We concentrate on the beam distribution which results from multi-turn correlated painting which is the primary painting scheme in the present SNS design [1]. In this paper we describe only the space-charge effect which is explored both analytically and numerically. We also present time evolution studies of the full-intensity beam after the end of the multi-turn injection process. Additional effects which also influence stability of the beam distribution, such as painting schemes and bump functions, magnet errors and imperfection resonances, space-charge coupling resonances, choice of working point, etc., are reported elsewhere [1]-[9].

\section{ANALYSIS}

\subsection{Painting procedure}

Here we present the simplified analysis of a painting procedure [10], which also may be obtained as a particular case from a more general treatment [3]. We assume a pencil-like linac beam which is injected into the ring at coordinates $(x, y)=(a, b)$, while the closed orbit is at $(x, y)=(u, v)$. The betatron motion is then determined by

$$
x-u=(a-u) \cos \nu_{x} t, y-v=(b-v) \cos \nu_{y} t .
$$

Assuming that $u(t)$ and $v(t)$ vary slowly, we populate the $(\dot{x}, x),(\dot{y}, y)$ phase spaces with the distribution function

$$
\begin{aligned}
f= & \text { const } \cdot \delta\left(\frac{\dot{x}^{2}}{\nu_{x}^{2}}+(x-u)^{2}-(a-u)^{2}\right) \\
& \delta\left(\frac{\dot{y}^{2}}{\nu_{y}^{2}}+(y-v)^{2}-(b-v)^{2}\right),
\end{aligned}
$$

\footnotetext{
* Work supported by the US Department of Energy
}

which results in the following charge distribution

$$
\rho=\int_{0}^{\tau_{f}} \frac{d \tau / \pi^{2}}{\sqrt{\left[(a-u(\tau))^{2}-x^{2}\right]\left[(b-v(\tau))^{2}-y^{2}\right]}},
$$

where $\tau$ is the charge density per unit length. For the case of correlated painting, the functions $u$ and $v$ have the form

$$
u(\tau)=a(1-g(\tau)), v(\tau)=b(1-g(\tau)),
$$

where $g(\tau)$ can be any desired bump function. We note that one gets logarithmicaly infinite density (with an assumption of infinitely small linac beam size) along the lines $x / a= \pm y / b$, regardless of the choice of $g(\tau)$. For the choice $g(\tau)=\sqrt{\tau / \tau_{f}}$, one can easily obtain

$$
\rho(x, y)=\frac{2 \tau_{f}}{\pi^{2} a b} \ln \left(\chi+\sqrt{\chi^{2}-1}\right),
$$

where $\chi=\sqrt{1-y^{2} / b^{2}} / \sqrt{x^{2} / a^{2}-y^{2} / b^{2}}$, with lines of constant density being hyperbolas. The $(\dot{x}, x),(\dot{y}, y)$ phase space projections will be uniformly populated ellipses with semi-axis $\left(a / \nu_{x}, a\right),\left(b / \nu_{y}, b\right)$, respectively.

\subsection{Effect of space charge}

The analytic calculation of space-charge forces from the charge distribution given by Eq. 5 is difficult for the square beam profile resulting from the correlated painting. We thus assumed a comparable model with cylindrical geometry based on the correct behavior near $x=y=0$. Using $x=r \cos \theta, y=r \sin \theta$, we obtain

$$
\rho(r, \theta)=C\left[\ln \frac{2 R}{r}-\sum_{m=1}^{\infty} \frac{(-1)^{m-1}}{m} \cos 4 m \theta\right],
$$

where $C, R$ are to be determined later, and $0 \leq r \leq R$. The electric potential due to Eq. 6 can be written as

$$
\Phi(r, \theta)=\frac{C}{\epsilon_{0}}\left[F_{0}+\sum_{m=1}^{\infty} \frac{(-1)^{m-1}}{m} F_{m}(r) \cos 4 m \theta\right] .
$$

Solving Poisson's equation, we obtain

$$
\begin{gathered}
F_{0}=\frac{R^{2}}{4}\left\{\begin{array}{cc}
-\frac{r^{2}}{R^{2}}\left(1+\ln \frac{2 R}{r}\right)+1+\ln 2 & , \quad r \leq R \\
(\ln 4+3) \ln \frac{R}{r} & , \quad r \geq R
\end{array}\right\}, \\
F_{m}(r)=\frac{R^{2}}{16 m\left(4 m^{2}-1\right)} \\
\cdot\left\{\begin{array}{ccc}
\frac{-4 m r^{2}}{R^{2}}+(2 m+1) \frac{r^{4 m}}{R^{4 m}} & , \quad r \leq R \\
-(2 m-1) \frac{R^{4 m}}{r^{4 m}} & , & r \geq R
\end{array}\right\} .
\end{gathered}
$$


We then choose $R=2 a / \sqrt{\pi}$ to keep the total area the same. The constant $C=\tau /\left(2 a^{2}[1+\ln 4]\right)$ is then obtained so that the total charge per unit length is $\tau$. The fields can be now calculated from Eq. 7. Since the charge density has a strong dependence on $\theta$ we observe the tendency of the space-charge force to equalize the dependence on $\theta$ through $E_{\theta}$, which only arises from the space-charge force. As a result, one finds that the azimuthal force vanishes due to symmetry at $\theta=0, \pi / 4$, etc., and is strongest at $\theta=\pi / 8$, where it acts to reduce the singularity at $\theta=\pi / 4$. The above analysis can be used to estimate the characteristic time scale of the diffusion process. Using the fact that $F_{m}$ decreases rapidly as $m$ increases, we approximate it by the leading $m=1$ term. We then find the time scale of the diffusion process in the azimuthal direction to be of the order of only a few turns in the ring. To estimate the radial diffusion one needs a more accurate conversion to cylindrical geometry with a subsequent analysis using the VlasovPoisson equation. However, due to the small tune depression we expect this diffusion to give only a modest growth (a few percent) in the beam radius. Thus, we explore the radial diffusion numerically in the next section.

\section{NUMERICAL SIMULATION}

\subsection{Beam distribution due to painting}

For the simulations presented in this paper we used the ORBIT code [11]. These studies were later extended taking into account the effect of magnet errors using the SNS simulation package based on the Unified Accelerator Libraries environment [8]. In the case of correlated painting, the beam is painted to a square shape; this results in a high density distribution along the diagonals which is in good agreement with the analytic prediction given by Eq. 5. Figure 1 shows a 2-D density plot (without space charge) for the square-root orbit bump function $g(\tau)=\sqrt{\tau / \tau_{f}}$. The inclusion of space charge leads to rapid azimuthal diffusion, in agreement with the analytic estimates. Figure 2 shows 2-D density plot (of the final distribution at the end of multi-turn injection) for the square-root bump function, with space charge included in simulations.

\subsection{Time evolution of distributions}

In our numerical simulations we need to optimize the bump function to find the best compromise between the target requirements, low beam loss and acceptable spacecharge tune spread. To satisfy the target requirement, which demands a density distribution close to uniform, we paint to a hollow distribution in the absence of space charge [4]. The required "hole" corresponds to a significant initial closed orbit offset from the injection foil. An example of a 1-D density profile with such painting is shown in Fig. 3 in the absence of space charge. Clearly, for such a significant closed orbit offset the square shape beam profile is no longer preserved due to a strong space-charge force at the beam boundary. The resulting transformation towards

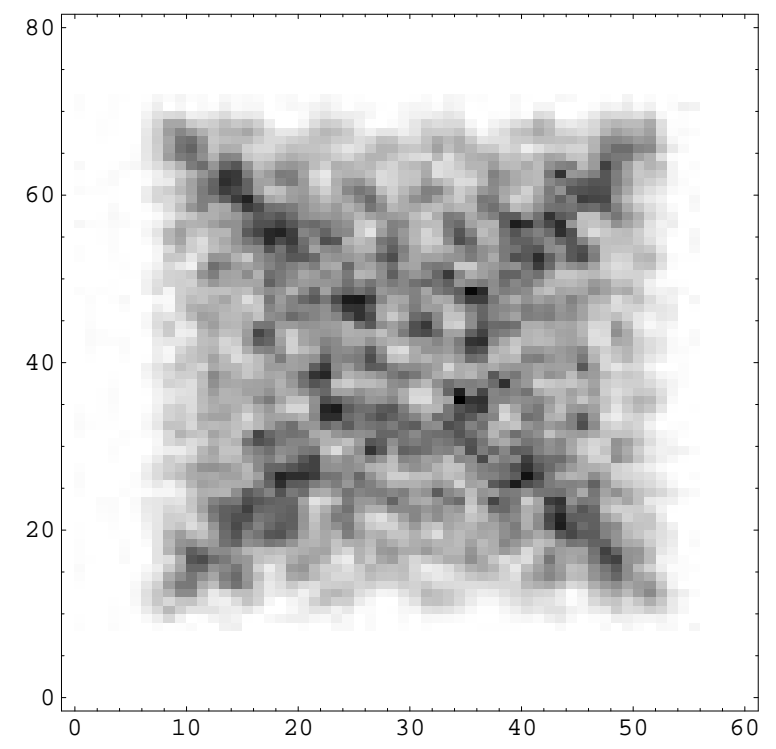

Figure 1: 2-D density plot (X-Y) for correlated painting with square-root bump function (without space charge).

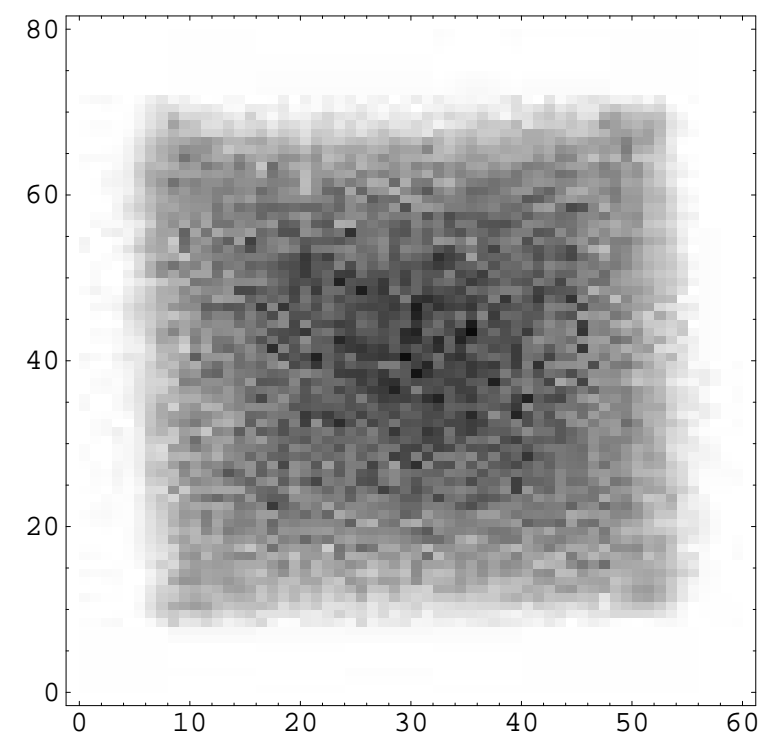

Figure 2: 2-D density plot (X-Y) for correlated painting with square-root bump function (with space charge).

a circular beam profile will be satisfactory as long as a density is close to uniform. Figure 4 shows the resulting beam profile after 1052 injection turns for the distribution which satisfies the target requirements. For other possible applications we also explore the feasibility of long time storage of a full intensity beam after multi-turn injection. Figure 5 shows time evolution of such a beam for the case of very small initial closed orbit offset ("small hole"). It clearly demonstrates the redistribution process associated with the square beam profile. However, for a "big hole" distribution, the beam profile is already circular by the end of injection which results in a subsequent smooth diffusion during additional storage time, as shown in Fig. 6. 


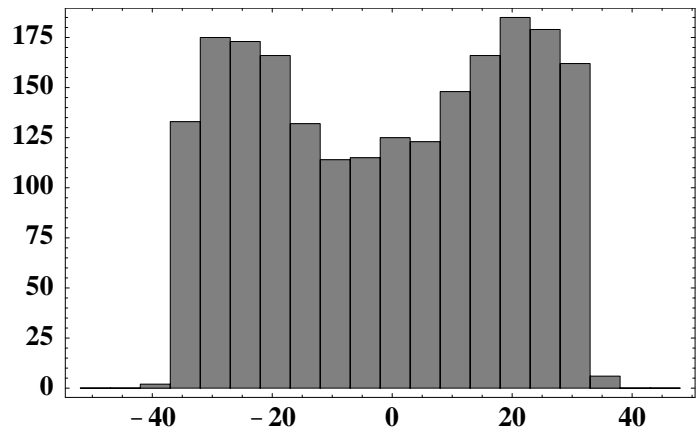

Figure 3: 1-D density profile in transverse direction. $\mathrm{X}$ axis - beam size in $\mathrm{mm}$, and $\mathrm{Y}$ axis - density in arbitrary units.

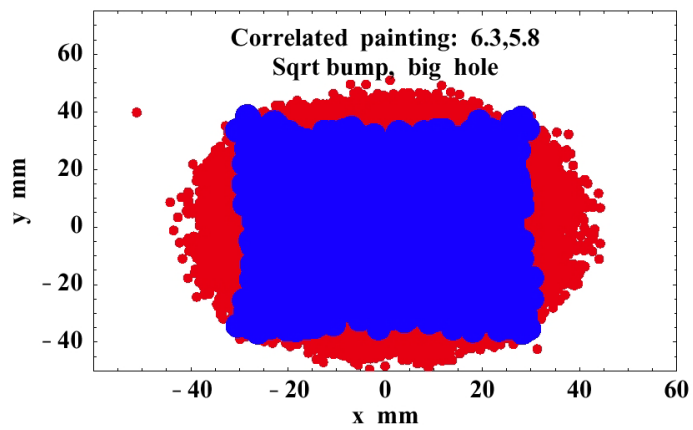

Figure 4: Beam profile with (red) and without (blue) space charge for bump function with significant initial closed orbit offset from injection foil ("big hole").

Another important issue for long time storage of a full intensity beams is the possibility of a parametric halo and associated emittance growth. Such a halo comes from a significant rms mismatch of the beam and is not expected during the multi-turn injection process [5], [12]. A parametric halo was for example observed in the simulations for the SNS lattice with close tunes $\nu_{x} \approx \nu_{y}$, using the KV distribution [13]. In the case of a realistic distribution described above and the split-tune case $\left(\nu_{x}, \nu_{y}\right)=(6.3,5.8)$ no such halo was observed. The resulting rms mismatch at the end of 1052 injection turns is small (of the order of a few percent). It stays at the same level during additional storage time shown in Figs. 5-6. Thus, the beam core is stable with some diffusion only in the tails. The radial diffusion shown in Figs. 5-6 becomes stronger when, for example, the magnet errors are included in simulations. However, the additional spreading is not dramatic as long as dangerous imperfection resonances are avoided [8], [9].

\section{ACKNOWLEDGMENT}

We thank the SNS team for dicussions and support.

\section{REFERENCES}

[1] J. Wei et al., Phys. Rev. ST AB, V. 3, 080101 (2000).

[2] J. Beebe-Wang et al., Proceedings of PAC99, p. 1743.

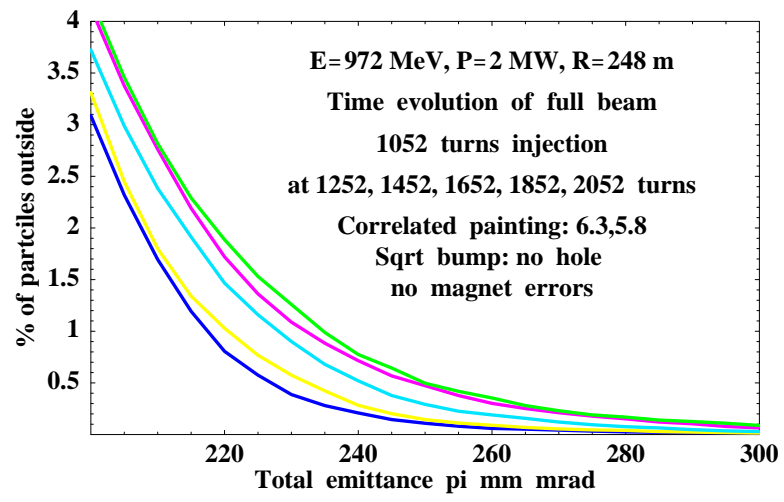

Figure 5: Distribution of beam halo at subsequent time steps (every 200 turns) after 1052-turn injection (from additional 200 turns (blue) to additional 1000 turns (green)). The case of a small initial closed orbit offset.

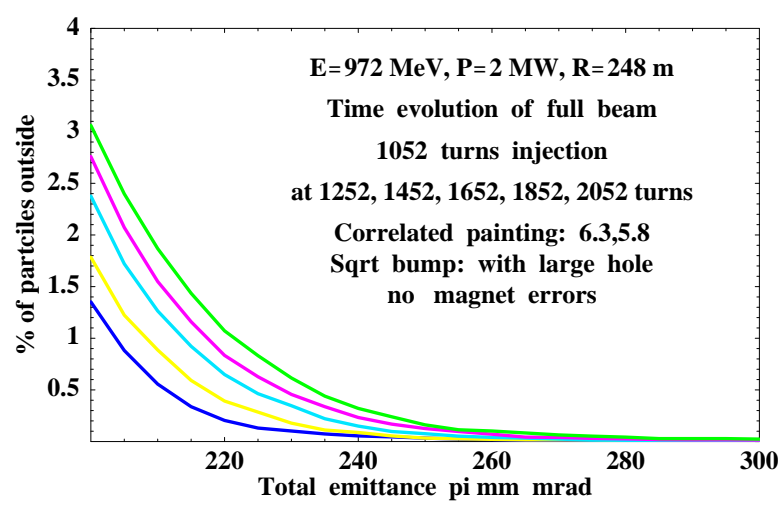

Figure 6: Distribution of beam halo at subsequent time steps (every 200 turns) after injection (from additional 200 turns (blue) to additional 1000 turns (green)). The case of large ("big hole") initial closed orbit offset.

[3] J. Beebe-Wang et al., Proceedings of EPAC'00 (Vienna, Austria, 2000), p. 1465.

[4] J. Beebe-Wang et al., as in Ref [3], p. 1286.

[5] A.V. Fedotov et al., as in Ref [3], p. 1289.

[6] A.V. Fedotov, J. Holmes and R.L. Gluckstern, "Instabilities of high-order beam modes driven by space-charge coupling resonances", submitted to Phys. Rev. ST AB (2001).

[7] A.V. Fedotov et al., "Excitation of resonances due to space charge and magnet errors in SNS ring", these proceedings.

[8] A.V. Fedotov, N. Malitsky and J. Wei, SNS/BNL Tech. Note 086 (2001).

[9] A.V. Fedotov, Y. Papaphilippou, J. Wei and J. Holmes, SNS/BNL Tech. Note 091 (2001).

[10] R.L. Gluckstern, "Painting Analysis", SNS/BNL Note, December 1999 (unpublished).

[11] J.D. Galambos et al., ORBIT Users Manual, SNS/ORNL/AP Tech. Note 011 (1999).

[12] A.V. Fedotov et al., 7th ICFA Workshop on Beam Halo and Scraping (Wisconsin, 1999), p.27.

[13] J.A. Holmes et al., Phys. Rev. ST AB, Vol. 2, 114202 (1999). 